\title{
Rosai-Dorfman Disease in Thoracic Spine: A Rare Case of Compression Fracture
}

\author{
Do Young Kim¹, Ji Hye Park ${ }^{2}$, Dong Ah Shin ${ }^{1}$, Seung $\mathrm{Yi}^{1}$, Yoon $\mathrm{Ha}^{1}$, \\ Do Heum Yoon ${ }^{1}$, Keung Nyun Kim ${ }^{1}$ \\ Departments of ${ }^{l}$ Neurosurgery, ${ }^{2}$ Pathology, Yonsei University College of Medicine, Seoul, Korea
}

Sinus histiocytosis with massive lymphadenopathy known as Rosai-Dorfman disease is characterized by painless bilateral cervical lymphadenopathy. Extranodal manifestations are uncommon and spinal involvement is rare. A 15-year-old man presented with intermittent midthoracic back pain only. He had no specific findings on neurologic examinations, hematologic and biochemical laboratory tests. Radiological examination of thoracic spine revealed collapse of T6 vertebrae with thoracic kyphosis and osteolytic lesion of T12 vertebra body. He underwent a removal of bone tumor, anterior reconstruction with mesh and pedicle screw fixation via posterior approach for pathologic confirmation and stabilization. Histopathologic study of the lesion revealed focal infiltration of large histiocytes showing emperipolesis. Immunochemistry stain of histiocytes was positive for CD68 and S-100 but negative for CD1a. This report presents a rare case and literature review of extranodal Rosai-dorfman disease in thoracic spine.

Key Words: Rosai-Dorfman disease $\cdot$ Extranodal involvement $\cdot$ Thoracic vertebra $\cdot$ Compression fracture

\section{INTRODUCTION}

Sinus histiocytosis with massive lymphadenopathy known as Rosai-Dorfman disease (RDD) was first described in 1965 and identified as a distinct clinicopathologic entity in $1969^{15}$. $\mathrm{RDD}$ is a rare histiocytic proliferative disorder of unknown etiology usually characterized by painless bilateral cervical lymphadenopathy ${ }^{3)}$. It typically affects young adults and adolescents. The common clinical findings are fever, lymph node enlargement, leukocytosis, neutrophila, elevation of erythrocyte sedimentation rate (ESR), hypergammaglobulinemia and weight loss. RDD is potentially systemic disease and may affect extranodal sites up to $43 \%$ of all RDD patients ${ }^{3)}$. The common extranodal sites include skin, upper respiratory tract, and bone $^{3)}$. Skeletal involvement as a sole manifestation of RDD is extremely rare, occurring in fewer than $2 \%$ of all $\mathrm{RDD}$

- Received: September 13, 2014 - Revised: September 20, 2014 - Accepted: September 22, 2014

Corresponding Author: Keung Nyun Kim, MD, PhD

Department of Neurosurgery, Severance Hospital, Yonsei University College of Medicine, 50-1, Yonsei-ro, Seodaemun-gu, Seoul 120-752, Korea

Tel: +82-2-2228-2151, Fax: +82-2-393-9979

E-mail: KNKIM@yuhs.ac

$\otimes$ This is an Open Access article distributed under the terms of the Creative Commons Attribution Non-Commercial License (http://creativecommons.org/ licenses/by-nc/3.0/) which permits unrestricted non-commercial use, distribution, and reproduction in any medium, provided the original work is properly cited. patients and arise most frequently in long bones such as tibia, femur, humerus, clavicle and bones of the hands ${ }^{2}$. We present a rare case of thoracic vertebrae compression fracture due to extranodal RDD without lymphadenopathy.

\section{CASE REPORT}

A 15-year-old man presented with intermittent midthoracic back pain for about 6 months after slip down during basketball. He had no other symptoms such as fever, chills, malaise and lymph node enlargement. There was no relevant past medical or surgical histories. There was no specific finding on neurological examination. Results of routine hematologic and biochemical laboratory tests include ESR and C-reactive protein (CRP) showed within reference ranges.

A plain radiological examination of thoracic spine revealed collapse of T6 vertebrae with thoracic kyphosis and osteolytic lesion of T12 vertebra body(Fig. 1A). Magnetic resonance (MR) image of thoracic spine demonstrated collapse of T6 vertebrae with mild cord compression. The lesions involving T6 and T12 vertebral body showed slightly high signal intensity in T2-weighted MR images (Fig. 1B-D) and homogenous enhancement in T1-weighted MR images after the administration of gadolinium (Fig. 1E-G). Preoperative computed tomography (CT) scan showed near complete collapse of T6 vertebra body and osteolytic lesion of T12 vertebra. (Fig. 

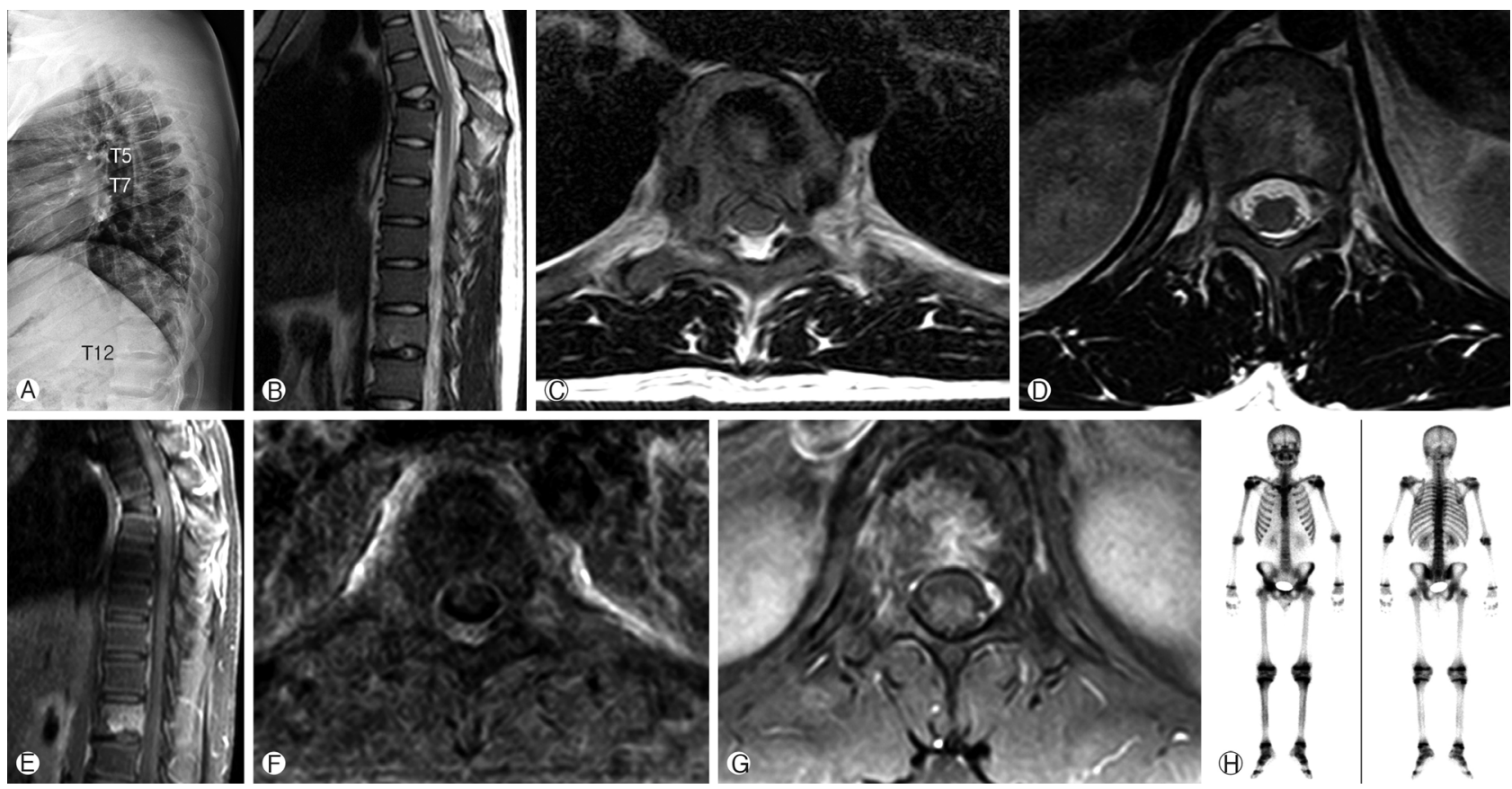

Fig. 1. Lateral view of thoracic spine on plain X-ray image (A) demonstrates compression fracture of T6 vertebrae and a well-defined osteolytic lesion on T12 vertebrae. The lesions involving T6 and T12 vertebral body shows slightly high signal intensity in T2- weighted MR images (B: sagittal, $C$ : axial of T6, D: axial of T12) and homogenous enhancement in T1-weighted MR images after the administration of gadolinium (E: sagittal, F: axial of T6, G: axial of T12), Increased tracer activity is shown at the T6, T12 vertebra and epiphysis in whole body bone scan $(\mathrm{H})$.
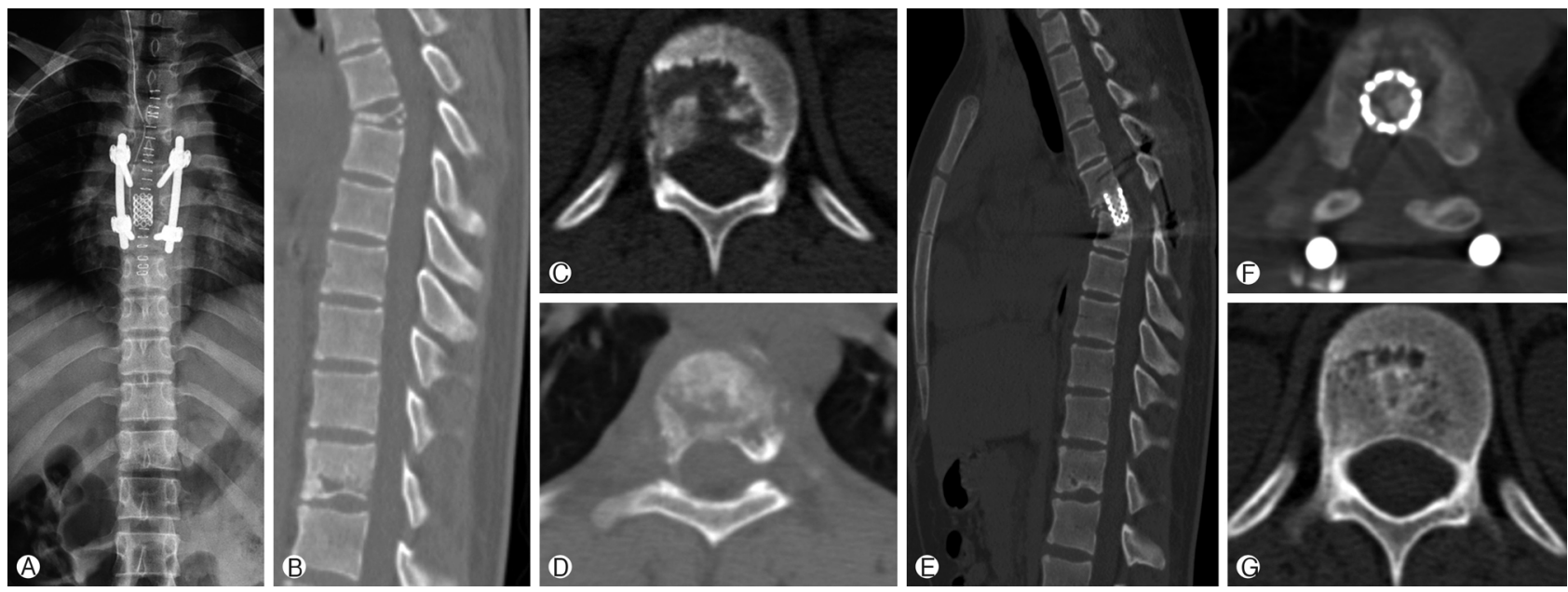

Fig. 2. Immediate postop operative X-ray image after removal of bone tumor, anterior interbody fusion and screw fixation (A). Preoperative CT scan of thoracic spine shows near complete collapse of T6 vertebra body and osteolytic lesion of T12 vertebra (B: sagittal, C: axial of T6, D: axial of T12). A follow-up CT scan of thoracolumbar spine at one year after operation presents good status of bone fusion on T6 vertebra and bone formation of T12 vertebra body compared with preoperative CT scan (E: sagittal, F: axial of T6, G: axial of T12).

2) Increased tracer activity was shown at the T6, T12 vertebra and epiphysis in whole body bone scan(Fig. $1 \mathrm{H}$ )

The patient underwent a total laminectomy of T6, left-sided facetectomy at T6/7, removal of bone tumor, anterior reconst- ruction with mesh and pedicle screw fixation at T5 and T7 for pathologic confirmation and stabilization. Immediate postoperative plain X-rays were shown in Fig. 2. He was discharged from hospital at postoperative 5 days without complica- 


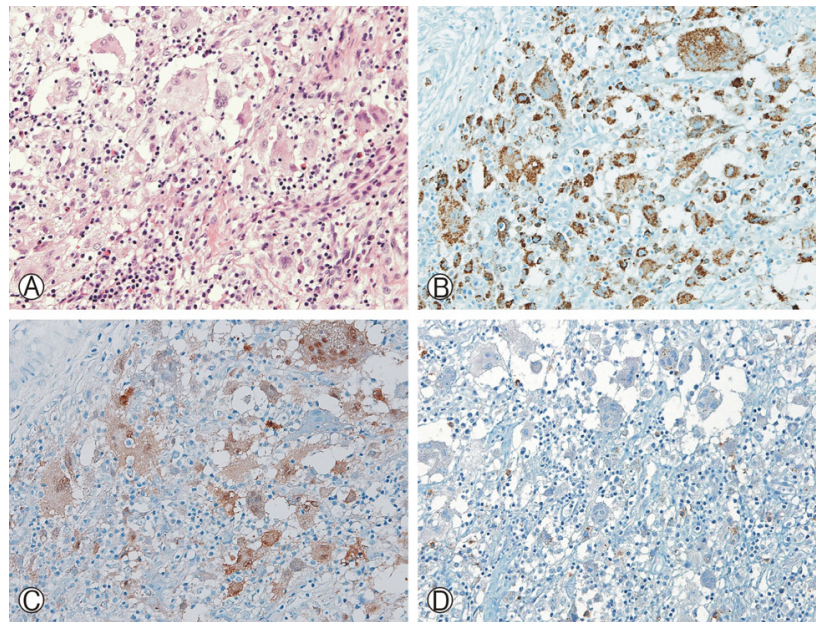

Fig. 3. Photomicrograph of the lesion demonstrates focal infiltration of large histiocytes showing emperipolesis (A). Immunochemistry stain shows that histiocytes are positive for CD68 (B) and S-100 (C), but negative for CDla (D).

tions and additional medications except some pain killers.

Histopathologic study of the lesion revealed fibro-osseous tissue showing focal infiltration of large histiocytes representing emperipolesis. These histiocytes were positive for CD68 and S-100, but negative for CD1a in immunochemistry study, that is compatible for RDD (Fig. 3).

A follow-up CT scan of thoracolumbar spine at one year after operation presented good status of bone fusion on T6 vertebra and bone formation of T12 vertebra body compared with preoperative CT scan that could be considered self-regression (Fig. 2).

\section{DISCUSSION}

The present case is a rare example of compression fracture caused by isolated extranodal RDD involved the thoracic spine without lymphadenopathy. RDD typically affects the bilateral cervical lymph nodes causing painless lymphadenopathy characterized by fever, lymph node enlargement, leukocytosis, neutrophila, elevation of ESR, hypergammaglobulinemia and weight loss. Most patients with RDD are in their second or third decade of life and there is predominant in males and African-Americans $^{3)}$. The etiology of RDD remains unknown; however it is implied that dysfunction of the immune system and an autoimmune process or viral infection such as Epsteine-Barr virus and human herpes virus type 6 might be the causative factors in some former studies, ${ }^{7,9,10)}$.

In general, extranodal manifestation without involvement of cervical lymph nodes is uncommon. The common extranodal sites include skin, upper respiratory tract, and bone ${ }^{3)}$.
Skeletal involvement as a sole manifestation of RDD is extremely rare, occurring in fewer than $2 \%$ of all RDD patients and usually occurs in the long bones such as tibia, femur, humerus, clavicle and bones of the hands or skull, and is multiple. Some cases that involved spine were reported in former studies. Some cases of RDD with intraspinal involvement in the form of intradural or epidural mass lesions causing neurologic deficit due to spinal cord compression ${ }^{1,8,16}$, and vertebra body involvement ${ }^{4,5,14)}$ were reported. Compression fracture due to spinal involvement of RDD had not been reported to the best our knowledge.

$\mathrm{RDD}$ is a rare diagnosis with a variety of imaging manifestations $^{13)}$, so usually requires histology for diagnosis. Skeletal lesions of RDD are typically intramedullary osteolytic with either poorly or sharply defined margins and are rarely sclerotic, that can be confused radiographically with Langerhans cell histiocytosis $(\mathrm{LCH})$, eosinophilic granuloma or other lymphocyte proliferative diseases, ${ }^{3,11)}$. The lesion presents low or isosignal intensity on T1- and T2-weighted MR images and intense homogenous enhancement with contrast in most cases, but MR images can be various ${ }^{1)}$. Spinal Rosai-Dorfman disease similarly tends to be confused with meningioma when presenting as dural disease or as metastatic disease of another cause when presenting as vertebral body disease ${ }^{13}$. The differential diagnosis of a primary bone Rosai-Dorfman disease includes more common lesions, such as bacterial osteomyelitis, fungal infections such as histoplasmosis and Langerhans cell histiocytosis. Special studies such as histochemistry, immunohistochemistry, and microbiologic cultures can be very useful to reach the correct diagnosis.

$\mathrm{LCH}$ is a close differential diagnosis both on radiology and histology. Both histiocytes of RDD and $\mathrm{LCH}$ are positive for CD68 and S100 in immunochemistry but histiocytes of RDD are negative for CD1a. RDD has a typical finding in histopathologic studies, that is emperioplesis; a phenomenon of phagocytosis of intact lymphocytes, plasma cells, erythrocytes or neutrophils. Langerhans cell histiocytosis does not exhibit this phenomenon. So, immunochemical characteristic of the lesions helps to determine the final diagnosis.

Natural history of RDD is uncertain but self-limiting and regresses spontaneously. Previous studies for treatment of RDD concluded that most patients with RDD do not require specific therapy and can expect spontaneous regression ${ }^{6,12)}$. If the vital organ is compressed or other life threatening manifestations are existed due to direct nodal or extranodal involvement, surgical debulking and/or radiotherapy can be consid$\operatorname{ered}^{12)}$. Other treatment modalities such as chemotherapy including methotrexate and 6-Mercaptopurin, high-dose alphainterferon, steroid or antiviral drugs had been attempted for treatment of RDD. Standardized guidelines for surgical treat- 
ment have not yet been established nor have the indications for a surgical approach. The performed operative technique was not different from that used to treat thoracic vertebrae fractures. For this case, it is widely accepted that the goal of surgery for thoracic compression fractures is decompression of the neural elements, correction of spinal deformities, and fusion with stabilization. We identified bone formation of T12 vertebra body in the follow-up CT scan of thoracolumbar spine at one year after operation, that could be considered to be resolved spontaneously.

\section{CONCLUSION}

Skeletal involvement of RDD is unusual and sole manifestation of spine vertebra is extremely rare. If the young adult or adolescent have compression of vertebra body, RDD can be considered as our case. Because of its rarity, various clinical manifestations and radiologic findings, it was difficult to differentiate from other disease such as Langerhans cell histiocytosis or eosinophilic granuloma on radiologic study alone, so pathologic confirmation was needed. As some former reports did, we could identify that RDD generally has benign clinical course and can be resolved itself.

\section{REFERENCES}

1. Abou-Zeid AH, Herwadkar A, du Plessis D, Gnanalingham KK: Isolated extradural Rosai-Dorfman disease of the thoracic spine: a rare cause of spinal cord compression: case report. Neurosurgery 67:E514-515, 2010

2. Demicco EG, Rosenberg AE, Bjornsson J, Rybak LD, Unni KK, Nielsen GP: Primary Rosai-Dorfman disease of bone: a clinicopathologic study of 15 cases. Am J Surg Pathol 34:1324-1333, 2010

3. Foucar E, Rosai J, Dorfman R: Sinus histiocytosis with massive lymphadenopathy (Rosai-Dorfman disease): review of the entity. Semin Diagn Pathol 7:19-73, 1990

4. Gupta P, Babyn P: Sinus histiocytosis with massive lymphaden- opathy (Rosai-Dorfman disease): a clinicoradiological profile of three cases including two with skeletal disease. Pediatr Radiol 38:721-728; quiz 821-722, 2008

5. Jing X, McHugh JB, Pu RT: Fine-needle aspiration cytology of Rosai-Dorfman disease of bone. Diagn Cytopathol 36:516518, 2008

6. Komp DM: The treatment of sinus histiocytosis with massive lymphadenopathy (Rosai-Dorfman disease). Semin Diagn Pathol 7:83-86, 1990

7. Luppi M, Barozzi P, Garber R, Maiorana A, Bonacorsi G, Artusi T, et al: Expression of human herpesvirus- 6 antigens in benign and malignant lymphoproliferative diseases. Am J Pathol 153: 815-823, 1998

8. Ma J, Xiao J, Wang L: Extranodal Rosai-Dorfman disease with multilevel lumbar spinal lesions. J Neurosurg Spine 9:55-57, 2008

9. McPherson CM, Brown J, Kim AW, DeMonte F: Regression of intracranial rosai-dorfman disease following corticosteroid therapy. Case report. J Neurosurg 104:840-844, 2006

10. Middel P, Hemmerlein B, Fayyazi A, Kaboth U, Radzun HJ: Sinus histiocytosis with massive lymphadenopathy: evidence for its relationship to macrophages and for a cytokine-related disorder. Histopathology 35:525-533, 1999

11. Miyake M, Tateishi U, Maeda T, Arai Y, Sugimura K, Hasegawa T: Extranodal Rosai-Dorfman disease: a solitary lesion with soft tissue reaction. Radiat Med 23:439-442, 2005

12. Pulsoni A, Anghel G, Falcucci P, Matera R, Pescarmona E, Ribersani M, et al: Treatment of sinus histiocytosis with massive lymphadenopathy (Rosai-Dorfman disease): report of a case and literature review. Am J Hematol 69:67-71, 2002

13. Raslan OA, Schellingerhout D, Fuller GN, Ketonen LM: RosaiDorfman disease in neuroradiology: imaging findings in a series of 10 patients. AJR Am J Roentgenol 196: W187-193, 2011

14. Robert EG, Fallon KB, Tender GC: Isolated Rosai-Dorfman disease of the sacrum. Case illustration. J Neurosurg Spine 4: 425, 2006

15. Rosai J, Dorfman RF: Sinus histiocytosis with massive lymphadenopathy. A newly recognized benign clinicopathological entity. Arch Pathol 87:63-70, 1969

16. Wang Y, Gao X, Tang W, Jiang C: Rosai-Dorfman disease isolated to the central nervous system: a report of six cases. Neuropathology 30:154-158, 2010 